\title{
Spin-Seebeck effect on the surface of a topological insulator due to nonequilibrium spin-polarization parallel to the direction of thermally driven electronic transport
}

\author{
Po-Hao Chang, ${ }^{1}$ Farzad Mahfouzi, ${ }^{1}$ Naoto Nagaosa, ${ }^{2,3}$ and Branislav K. Nikolic ${ }^{1,2, \text { * }}$ \\ ${ }^{1}$ Department of Physics and Astronomy, University of Delaware, Newark, DE 19716-2570, USA \\ ${ }^{2}$ RIKEN Center for Emergent Matter Science (CEMS), Wako, Saitama 351-0198, Japan \\ ${ }^{3}$ Department of Applied Physics, University of Tokyo, Tokyo 113-8656, Japan
}

\begin{abstract}
We study the transverse spin-Seebeck effect (SSE) on the surface of a three-dimensional topological insulator (TI) thin $\mathrm{lm}$, such as $\mathrm{Bi}_{2} \mathrm{Se}_{3}$, which is sandwiched between two normal metal leads. The temperature bias $\Delta T$ applied between the leads generates surface charge current which becomes spin polarized due to strong spin-orbit coupling on the TI surface, with polarization vector acquiring a component $P_{x} \simeq 60 \%$ parallel to the direction of transport. When the third nonmagnetic voltage probe is attached to the portion of the TI surface across its width $L_{y}$, pure spin current will be injected into the probe where the inverse spin Hall effect (ISHE) converts it into a voltage signal $\left|V_{\text {ISHE }}\right|^{\max } / \Delta T \simeq 2.5 \mu \mathrm{V} / \mathrm{K}$ (assuming the $\mathrm{SH}$ angle of the $\mathrm{Pt}$ voltage probe and $L_{y}=1 \mathrm{~mm}$ ). The existence of predicted nonequilibrium spin polarization parallel to the direction of electronic transport and the corresponding electron-driven SSE crucially relies on orienting quintuple layers (QLs) of $\mathrm{Bi}_{2} \mathrm{Se}_{3}$ orthogonal to the TI surface and tilted by $45^{\circ}$ with respect to the direction of transport. Our analysis is based on the Landauer-Büttiker-type formula for spin currents in the leads of a multiterminal quantum-coherent junction, which is constructed by using nonequilibrium Green function formalism within which we show how to take into account arbitrary orientation of QLs via the self-energy describing coupling between semi-innite normal metal leads and the TI sample.
\end{abstract}

PACS numbers: 72.25.Dc, 72.15.Jf, 85.75.-d, 85.80.Fi

\section{INTRODUCTION}

The spin-Seebeck effect (SSE) is a recently observed phe- nomenon where spin current or spin accumulation is induced by a temperature gradient applied across a ferromagnetic material.12 . At rst sight, the SSE appears to be a counterpart of the traditional charge-Seebeck effect (CSE) where the temperature gradient across a conductor induces electrical current (in closed circuits) or voltage (in open circuits). However, the SSE has been observed in a surprisingly wide range of materials, including ferromagnetic insulators where the CSE does not exist. 112

In the so-called transverse SSE measurement geometry, illustrated in Fig. 1. a temperature gradient applied longitudinally over a strip of magnetic material induces a spin signal detected by measuring voltage $V_{\text {ISHE }}$ generated via the inverse spin Hall effect (ISHE) in the nonmagnetic metallic probe (such as $\mathrm{Pt}$ ) attached on top of the strip across its width. The voltage signal $V_{\text {ISHE }}$ is found to be approximately linear (in ferromagnetic metals and insulator ${ }^{2}$ ) or a hyperbolic sine (in ferromagnetic semiconductors ${ }^{3}$ ) function of the probe position in the longitudinal direction over the length of several millimeters. Since this is surprisingly long when compared to the usual electronic spin-dependent length scales, recent theories of the SSE have focused on the interplay of magnons and phonons out of equilibrium. [4.

On the other hand, the role of spin-polarized conduction electrons in SSE generation has been much less explored. The need for this has been prompted by the very recent experimenta ${ }^{5}$ unveiling of "giant" $V_{\text {ISHE }}$ (up to a

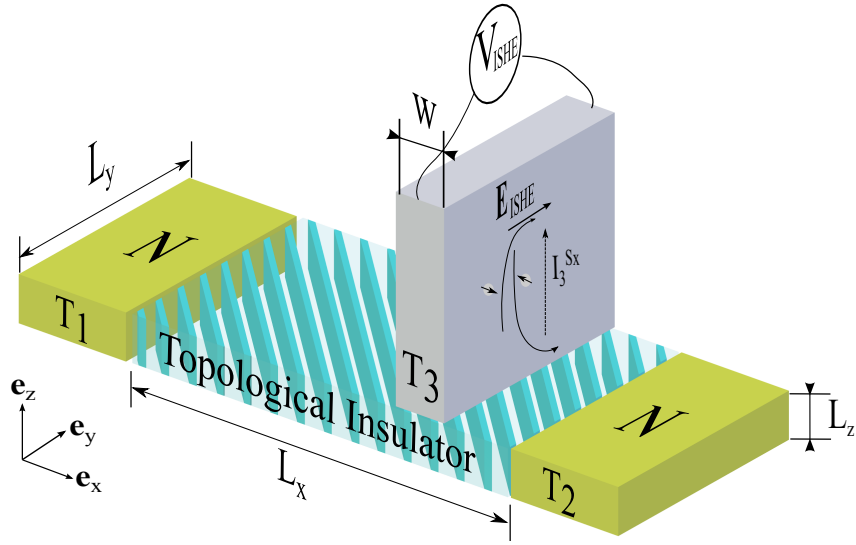

FIG. 1: (Color online) Schematic view of the three-terminal junc- tion where a thin $\mathrm{lm}$ of $\mathrm{Bi}_{2} \mathrm{Se}_{3}$ is attached to two nonmagnetic metallic leads kept at different temperatures $T_{1}>T_{2}$. The SSE voltage signal $V_{\text {ISHE }}$ is measured across the edges of the third nonmagnetic metallic lead attached to the top TI surface a function of its position along the $\mathrm{x}$ axis. This lead acts as voltage probe attached to a macroscopic reservoir at potential $V_{3}$ to ensure zero net charge current through it. We orient quintuple layers of $\mathrm{Bi}_{2} \mathrm{Se}_{3}$ at an angle of $45^{\circ}$ with respect to the direction ( $x$ axis) of electron propagation on the TI surface that is orthogonal to these layers.

thousand times larger than observed in measurements on magnetic materials ${ }^{2}$ ) in the transverse SSE setup where a nonmagnetic semiconductor InSb was placed in a large longitudinal (parallel to the temperature gradient) external magnetic eld. Aside from the magnitude of $V_{\text {ISHE }}$, which is speculated to arise in the interplay of spin-orbit 
coupling (SOC) and enhancement of the phonon drag contribution to both the spin- and charge-Seebeck coefcients for electrons pushed into the ultraquantum limit by the applied magnetic eld, another puzzle for SSE theories posed by Ref. 5 is that $V_{\mathrm{ISHE}}$ did not change sign under the reversal of the magnetic-eld direction.

Here we show that basic phenomenology of the experiment in Ref. 5 can be recreated without applying any external magnetic eld. The role of the magnetic eld was to spin polarize electrons in the direction of transport (by means of Zeeman splitting, further amplied by SOC in InSb), as well as to conne their spatial motion so that electrons spiral in the $y z$ plane (with cyclotron orbits that are quantized into Landau levels) as they translate along the $x$ axis. In order to generate the same spin polarization along the electron transport direction, we employ a thin $\mathrm{lm}$ of a recently discovered three- dimensional topological insulator (3D TI) material, such as $\mathrm{Bi}_{2} \mathrm{Se}_{3}$ assumed here, which is attached to three normal (i.e., nonmagnetic) metal (N) leads, as illustrated in Fig. 1 .

The 3D TI materials ${ }^{6}$ possess a usual band gap in the bulk, while hosting metallic surfaces whose lowenergy quasiparticles are massless Dirac fermions with spins locked to their momenta due to strong Rashba-type SOC ${ }^{77} \mathrm{In}$ particular, $\mathrm{Bi}_{2} \mathrm{Se}_{3}$ realization of TI is a strongly anisotropic material composed of quintuple layers (QLs) of $\mathrm{Bi}$ and Se atoms, where one QL consists of three Se layers strongly bonded to two Bi layers in between. ${ }^{[6}$ While $\mathrm{Bi}_{2} \mathrm{Se}_{3}$ is always unintentionally $n$-type doped by Se vacancies, charge carriers in the bulk of films of thickness $\lesssim 10 \mathrm{~nm}$ can be completely removed by a gate electrode. 8

One of the key effects 10 that 3D TIs bring into spintronics is nonequilibrium spin density in the direction transverse to injected unpolarized charge current, which is much larger $\frac{12}{12}$ than in the case $e^{14}-\frac{16}{16}$ of two-dimensional electron gases (2DEGs) with the Rashba SOC. Our first principal result [see Figs. 3(a) and 3(b)] demonstrates that additional component of nonequilibrium spin density and polarization can be induced in the direction parallel to injected charge current, on the proviso that QLs are oriented as shown in Fig. 1. Our second principal result [see Figs. 4(a)-(c)] shows that this indeed makes possible non-zero SSE signal in three-terminal geometry of the junction depicted in Fig. 1.

The paper is organized as follows: In Sec. II we employ the nonequilibrium Green function (NEGF) formalism 17 to obtain the Landauer-Büttiker (LB-type) formula for spin currents in the leads of a multi-terminal quantumcoherent junction driven by both voltage bias and temperature bias in the linear-response regime. Section III explains our Hamiltonian model for the TI $\mathrm{lm}$, as well as the construction of the retarded GF for an open system TI + semi-innite $\mathrm{N}$ leads where the orientation of QLs shown in Fig. 1 is taken into account through the self-energy entering the retarded GF. In Sec. IV we ana- lyze the spin-polarization vector of the charge current, as well as charge conductance, for a TI lm attached to two N leads. In Sec. V $\mathrm{V}$ we predict the magnitude of the voltage signal generated across the third $\mathrm{N}$ lead in the threeterminal junction depicted in Fig. 1, while also contrasting its features with those of conventional charge and spin-dependent Seebeck coefcients that would be measured between terminals 1 and 2 in Fig. 1. We conclude in Sec. VI.

\section{SPIN CURRENTS IN MULTI-TERMINAL QUANTUM-COHERENT CONDUCTORS DRIVEN BY VOLTAGE AND TEMPERATURE BIASES}

We use the same units for the total charge $I_{p}=I_{p}^{\uparrow}+I_{p}^{\downarrow}$ and total spin $I_{p}^{S_{\alpha}}=I_{p}^{\uparrow}-I_{p}^{\downarrow}$ currents owing through lead $p$, which are constructed from spin-resolved charge currents $I_{p}^{\sigma}$ with the spin quantization axis for $\sigma=\uparrow, \downarrow$ chosen along $\mathbf{e}_{\alpha}$. There has been a lively debate ${ }^{18 \mid 19}$ in the literature on the proper derivation of the multi-terminal LB-type formula ${ }^{20}$ which connects spin current $I_{p}^{S_{\alpha}}$ owing through the semi-innite ideal (i.e., charge- and spininteraction-free) metallic lead $\mathrm{p}$ attached to a quantumcoherent conductor due to voltages $V_{p}$ applied at the external macroscopic reservoirs into which the leads terminate at innity. The debate was spurred by one of the early derivations,,$\frac{21}{, 19}$ using the traditional scattering matrix framework [19], which predicted unphysical $I_{p}^{S_{\alpha}} \neq 0$ in equilibrium $V_{p}=$ const.

Here we derive LB-type formula for spin currents driven by both voltage and temperature biases. We bypass the issue of unphysical equilibrium total spin currents ${ }^{18 \mid 19}$ by starting from the outset from a general NEGF-based expression for spin current in lead $p$ :

$$
I_{p}^{S_{\alpha}}=\frac{e}{h} \int d E \operatorname{Tr}\left\{\hat{\sigma}_{\alpha}\left[\boldsymbol{\Sigma}_{p}^{<}(E) \mathbf{G}^{>}(E)-\boldsymbol{\Sigma}_{p}^{>}(E) \mathbf{G}^{<}(E)\right]\right\} .
$$

This is actually the difference of spin-resolved charge currents given by the well-known MeirWingreen formula, $\frac{17}{17}$ where $\left(\hat{\sigma}_{x}, \hat{\sigma}_{y}, \hat{\sigma}_{z}\right)$ is the vector of the Pauli matrices. The two fundamental objects of the NEGF formalismthe retarded $\mathbf{G}(E)$ and the lesser $\mathbf{G}^{<}(E)=\mathbf{G}(E) \boldsymbol{\Sigma}^{<}(E) \mathbf{G}^{\dagger}(E)$ GFs - describe the density of available quantum states and how electrons occupy those states, respectively 17

In the elastic transport regime the lesser selfenergies, $\boldsymbol{\Sigma}_{p}^{<}(E)=i f_{p}(E) \boldsymbol{\Gamma}_{p}(E)$ and $\boldsymbol{\Sigma}^{<}=\sum_{p} \boldsymbol{\Sigma}_{p}^{<}(E)$, are express- ible in terms of the retarded self-energies $\boldsymbol{\Sigma}_{p}(E)$ using $\boldsymbol{\Gamma}_{p}=i\left[\boldsymbol{\Sigma}_{p}(E)-\boldsymbol{\Sigma}_{p}^{\dagger}(E)\right]$ and $f_{p}(E)$ as the Fermi distribution of electrons within the reservoirs. This makes it possible to rewrite Eq. (1) for the total spin current in lead $p$ as 


$$
I_{p}^{S_{\alpha}}=\frac{e}{h} \sum_{q} \int d E \operatorname{Tr}\left[\hat{\sigma}_{\alpha} \boldsymbol{\Gamma}_{q}(E) \mathbf{G}(E) \boldsymbol{\Gamma}_{p}(E) \mathbf{G}^{\dagger}(E)\right]\left\{f_{p}(E)-f_{q}(E)\right\}
$$

By expanding $f_{p}(E)-f_{q}(E)$ to linear order in $T_{p}-T_{q}$ and $V_{p}-V_{q}$, we nally get the desired multiterminal LB-type formula for spin current driven by both temperature and voltage biases in the linear-response regime:

$$
I_{p}^{S_{\alpha}}=\frac{e^{2}}{h} \sum_{q} \int d E \operatorname{Tr}\left[\hat{\sigma}_{\alpha} \boldsymbol{\Gamma}_{q}(E) \mathbf{G}(E) \boldsymbol{\Gamma}_{p}(E) \mathbf{G}^{\dagger}(E)\right]\left\{\frac{\partial f}{\partial E}\left[\frac{E-E_{F}}{e T}\left(T_{p}-T_{q}\right)-\left(V_{p}-V_{q}\right)\right]\right\} .
$$

Note that the usual expression ${ }^{20}$ for the total charge current $I_{p}$ in lead $p$ is the same as Eq. (3), except that $\hat{\sigma}_{\alpha} \mapsto \hat{\sigma}_{0}$ where $\hat{\sigma}_{0}$ is the unit $2 \times 2$ matrix.

Applying Eq. (3) to the three-terminal junction in Fig.. 1 gives

$$
I_{p}^{S_{\alpha}}=\frac{e^{2} L_{y}}{2 \pi h} \sum_{q=1}^{3} \iint d E d k_{y} \operatorname{Tr}\left[\hat{\sigma}_{\alpha} \boldsymbol{\Gamma}_{q}(E) \mathbf{G}(E) \boldsymbol{\Gamma}_{p}(E) \mathbf{G}^{\dagger}(E)\right]\left\{\frac{\partial f}{\partial E}\left[\frac{E-E_{F}}{e T}\left(T_{p}-T_{q}\right)-\left(V_{p}-V_{q}\right)\right]\right\},
$$

where we use $T_{1}>T_{2}, T_{3}(x)=T_{1}-x\left(T_{1}-T_{2}\right) / L_{x}$, and $V_{1}=V_{2} \neq V_{3}$. By imposing the condition $I_{p}=0$ in one of the leads, such as lead $p=3$ in Fig. 1, the linear system of equations in Eq. (4) can be solved to nd voltage $V_{3}$ that has to be applied to convert this lead into a voltage probe employed in SSE experiments.

Using the spin-dependent transmission function $\mathcal{T}_{21}^{\alpha}(E)=\operatorname{Tr}\left[\hat{\sigma}_{\alpha} \boldsymbol{\Gamma}_{2}(E) \mathbf{G}(E) \boldsymbol{\Gamma}_{1}(E) \mathbf{G}^{\dagger}(E)\right]$ of the twoterminal version of junction in Fig. 1 w we can compute the following integrals $\$ 22 \mid 23$

$$
K_{n}^{\alpha}(\mu)=\frac{1}{h} \int_{-\infty}^{\infty} d E \mathcal{T}_{21}^{\alpha}(E)\left(E-E_{F}\right)^{n}\left(-\frac{\partial f}{\partial E}\right)
$$

which yield the three spin-dependent Seebeck coefficients $S_{\text {spin }}^{\alpha}=K_{1}^{\alpha} /\left(e T K_{0}^{\alpha}\right)$ for the chosen Pauli matrix $\hat{\sigma}_{\alpha}$, or the charge-Seebeck coefficient $S_{\text {charge }}=K_{1} /\left(e T K_{0}\right)$ when $\hat{\sigma}_{\alpha} \mapsto \hat{\sigma}_{0}$.

\section{HAMILTONIAN AND RETARDED GREEN FUNCTION FOR MULTI-TERMINAL TI-BASED JUNCTIONS}

The junction in Fig. 1 is modeled on the simple cubic lattice with lattice spacing $a$, which is assumed to be periodically repeated in the $y$-direction. The TI thin film has finite length $L_{x}$, while it is sufficiently thick $L_{z}=30 a$ to ensure no coupling between the top and the bottom metallic surfaces which penetrate as evanescent states into the bulk of the TI film and whose overlap would open a minigap at the Dirac point (DP) in ultrathin films.24 The TI thin film is described using the minimal tight- binding Hamiltonian with four orbitals per site ${ }^{25}$

$$
\begin{aligned}
\mathbf{H}_{\mathrm{TI}}= & \sum_{n, k_{y}}\left(\mathbf{c}_{n, k_{y}}^{\dagger}\left[\mathbf{M}_{0}+C \mathbf{1}+\mathbf{T}_{y} e^{i k_{y} a}+\mathbf{T}_{y}^{\dagger} e^{-i k_{y} a}\right] \mathbf{c}_{n, k_{y}}\right) \\
& +\sum_{n, k_{y}, \alpha=x, z}\left(\mathbf{c}_{n, k_{y}}^{\dagger} \mathbf{T}_{\alpha} \mathbf{c}_{n+\mathbf{e}_{\alpha}, k_{y}}+\text { H.c. }\right)
\end{aligned}
$$

where $\mathbf{T}_{\alpha}=B \hat{\sigma}_{z} \otimes \sigma_{0}-i A \hat{\sigma}_{x} \otimes \hat{\sigma}_{\alpha} / 2 ; \quad \mathbf{M}_{0}=(M-$ $6 B) \hat{\sigma}_{z} \otimes \hat{\sigma}_{0} ; \hat{\sigma}_{0}$ is the unit $2 \times 2$ matrix; and $\mathbf{1}=\hat{\sigma}_{0} \otimes \hat{\sigma}_{0}$. Here $\mathbf{c}_{n, k_{y}}=\left(\hat{c}_{+\uparrow}, \hat{c}_{+\downarrow}, \hat{c}_{-\uparrow}, \hat{c}_{-\downarrow}\right)^{T}$ annihilates electron in different orbitals on site $n$ with the transverse momentum $k_{y}$. The numerical values of the parameters are chosen as: $M=0.3 \mathrm{eV} ; A=0.5 \mathrm{eV}$; and $B=0.25 \mathrm{eV}$. The bottom of the band of TI is shifted by $C=3.0 \mathrm{eV}$.

When applying the in-plane external magnetic field $B_{x}$, the Zeeman term changes to $\mathbf{M}_{0} \mapsto \mathbf{M}_{0}+\Delta \hat{\sigma}_{0} \otimes \hat{\sigma}_{x}$ where $\Delta=g_{\|} \mu_{B} B_{x}$. We also apply an additional Zeeman term $\Delta \hat{\sigma}_{0} \otimes \hat{\sigma}_{z}$ with $\Delta=0.5 \mathrm{eV}$ to the bottom TI surface in order to split its Dirac cone and block current through it.

The semi-infinite $\mathrm{N}$ leads made of nonmagnetic metallic material are described by a tight-binding Hamiltonian with a single orbital per site

$$
\begin{aligned}
\mathbf{H}_{N}= & \sum_{n, \sigma, k_{y}} \varepsilon_{n, k_{y}} \hat{c}_{n \sigma, k_{y}}^{\dagger} \hat{c}_{n \sigma^{\prime}, k_{y}} \\
& -\gamma \sum_{n, \sigma, k_{y}, \alpha=x, z}\left(\hat{c}_{n \sigma, k_{y}}^{\dagger} \hat{c}_{n+\mathbf{e}_{\alpha}, \sigma, k_{y}}+\text { H.c. }\right) .
\end{aligned}
$$

where the operators $\hat{c}_{n \sigma}^{\dagger}\left(\hat{c}_{n \sigma}\right)$ create (annihilate) electron with spin $\sigma$ on site $n$ with the transverse momentum $k_{y}$. The kinetic energy $\varepsilon_{n, k_{y}}=-2 \gamma \cos k_{y} a$ is equivalent to an increase in the on-site energy, and the nearest neighbor hopping is set at $\gamma=1.0 \mathrm{eV}$.

The evaluation of Eq. (4) of relies crucially on the construction of the proper coupling matrix $\boldsymbol{\tau}$ between $\hat{H}_{\mathrm{TI}}$ in Eq. (6) and $\hat{H}_{N}$ in Eq. (7) since $\tau$ enters into the retarded 
GF. For example, the Hamiltonian of the composite system semi-infinite-N-lead-1 + TI-thin-film is given by

$$
\mathbf{H}_{N+\mathrm{TI}}=\left(\begin{array}{cc}
\mathbf{H}_{N} & \boldsymbol{\tau}_{1} \\
\boldsymbol{\tau}_{1}^{\dagger} & \mathbf{H}_{\mathrm{TI}}
\end{array}\right) .
$$

The retarded GF of the TI film alone, viewed as an open quantum system, is defined by $17 \mid 20$

$$
\mathbf{G}(E)=\left[E-\mathbf{H}_{\mathrm{TI}}-\boldsymbol{\Sigma}_{1}(E)\right]^{-1} .
$$

Here the retarded self-energy introduced by the semiinfinite $\mathrm{N}$ lead 1 is

$$
\boldsymbol{\Sigma}_{1}(E)=\boldsymbol{\tau}_{1}^{\dagger} \cdot \mathbf{g}(E) \cdot \boldsymbol{\tau}_{1},
$$

and $(\eta$ is positive infinitesimal)

$$
\mathbf{g}(E)=\left[E+i \eta-\mathbf{H}_{N}\right]^{-1},
$$

is the retarded $\mathrm{GF}^{17 / 20}$ of $\mathrm{N}$ lead 1. The same procedure would be repeated when more than one $\mathrm{N}$ lead is attached to the TI thin film to get

$$
\mathbf{G}(E)=\left[E-\mathbf{H}_{\mathrm{TI}}-\boldsymbol{\Sigma}_{1}(E)-\boldsymbol{\Sigma}_{2}(E)-\boldsymbol{\Sigma}_{3}(E)\right]^{-1}
$$

for the three-terminal junction in Fig. 1 .

The conventionally assumed identical orientation of spin (i.e., expectation value of the spin operator) on $\mathrm{Bi}$ and Se sublattices, where spin on both sublattices follows "helical" texture shown in Fig. 3 for Bi sublattice, is valid only on the (111) surface of the TI crystal that coincides with the plane of the QL. For TI surface other than (111), the spin operators for electrons residing on the Bi and Se sublattices are inequivalent. ${ }^{26}$ Nevertheless, for interpreting spin- and angular- resolved photoemission spectroscopy (spin-ARPES) experiments 6 or for attaching the TI sample to $\mathrm{N}$ leads, where photoelectrons or electrons injected or absorbed from $\mathrm{N}$ leads do not carry a sublattice index, it is advantageous to select the standard relation between the Pauli matrices and the spin operator, $\hat{\mathbf{S}}=\hbar \hat{\boldsymbol{\sigma}} / 2$.

The spinors $u_{\mathrm{Bi}}$ and $u_{\mathrm{Se}}$, associated with each sublattice when inequivalent spin operators are used,

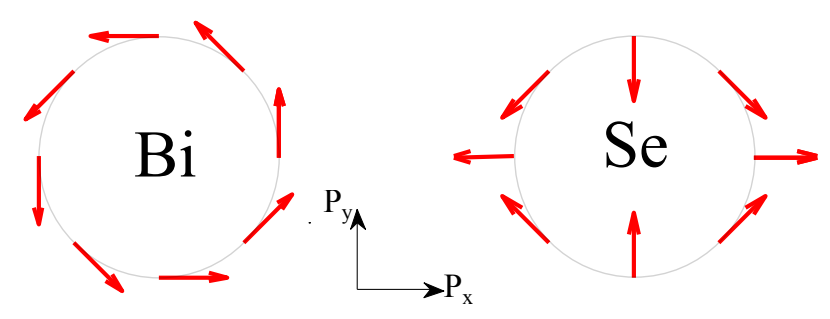

FIG. 2: (Color online) The equilibrium expectation values of the spin operator for surface-state electrons on the $\mathrm{Bi}$ and Se sublattices as a function of the in-plane momentum $\mathbf{p}=\left(p_{x}, p_{y}\right)$. The $\mathrm{Bi}_{2} \mathrm{Se}_{3}$ crystal is assumed to fill the halfspace $z<0$, so that its infinite surface in the $x y$-plane is orthogonal to its QLs that are also oriented perpendicularly to the unit vector $\mathbf{n}=\left(\mathbf{e}_{x}+\mathbf{e}_{y}\right) / \sqrt{2}$, as illustrated in Fig. 11 have to be unitarily transformed ${ }^{26}$ to $u_{\mathrm{Bi}} \mapsto u_{\mathrm{Bi}}$ and $u_{\mathrm{Se}} \mapsto i(\hat{\boldsymbol{\sigma}} \cdot \mathbf{n}) u_{\mathrm{Se}}$. Here $\mathbf{n}$ is the unit vector normal to the QL. This specifies $2 \times 4$ coupling matrices for each $\mathrm{N}$ lead $p=1-3$ as

$$
\boldsymbol{\tau}_{p}=\left(\begin{array}{cccc}
t_{\mathrm{Bi}} & 0 & i n_{z} t_{\mathrm{Se}} & \left(i n_{x}+n_{y}\right) t_{\mathrm{Se}} \\
0 & t_{\mathrm{Bi}} & \left(i n_{x}-n_{y}\right) t_{\mathrm{Se}} & -i n_{z} t_{\mathrm{Se}}
\end{array}\right) .
$$

For the setup in Figs. 1 and 2 we use $\mathbf{n}=(110)$ in Eq. (13). In addition, the hopping parameters between orbitals in the $\mathrm{N}$ leads and those on the $\mathrm{Bi}$ or Se sublattice are chosen as: $t_{\mathrm{Bi}}=0.4 \mathrm{eV}$ for leads 1 and 2 ; $t_{\mathrm{Bi}}=0.45 \mathrm{eV}$ for lead $3 ; t_{\mathrm{Se}}=0.8 \mathrm{eV}$ for leads 1 and 2 ; and $t_{\mathrm{Se}}=0.9 \mathrm{eV}$ for lead 3 .

\section{NONEQUILIBRIUM SPIN POLARIZATION IN TWO-TERMINAL TI-BASED JUNCTIONS}

When unpolarized charge current is injected from $\mathrm{N}$ lead 1, the ensemble of outgoing spins in $\mathrm{N}$ lead 2 of $\mathrm{N}_{1} / \mathrm{TI} / \mathrm{N}_{2}$ two-terminal junction is characterized by the spin density matrix $\hat{\rho}_{\text {spin }}^{\text {out }}=\frac{1}{2}(1+\mathbf{P} \cdot \hat{\boldsymbol{\sigma}})$ whose polarization vector is given by $P_{\alpha}=I_{p}^{S_{\alpha}} / I_{p}$ for such setup. We first demonstrate in Fig. 3(a) that an unpolarized charge current injected from $\mathrm{N}$ lead 1 into the top surface of a two-terminal junction (i.e., when the third $\mathrm{N}$ lead in Fig. 1 is removed) will exit into $\mathrm{N}$ lead 2 with non-zero spin-polarization vector $\mathbf{P}=\left(P_{x}, P_{y}, P_{z}\right)$ which includes a component $P_{x} \neq 0$ in the direction of transport. Since Dirac fermions on the opposite surfaces of TI have opposite chiralities, which generates opposite spinpolarization for currents flowing through the top and bottom surface that would cancel in the total current in $\mathrm{N}$ lead 2, we block transport through the bottom surface by introducing an energy gap into its Dirac cone (e.g., due to coating by magnetic film ${ }^{9}$ ). Figure $3(\mathrm{~b})$ reveals that current spin-polarization is established on a very short length scale of $\simeq 10$ lattice spacings, so that this mechanism can operate near or under the contacts with $\mathrm{N}$ leads even in the presence of inevitable spin or charge dephasing mechanisms (the spin dephasing time for the in-plane spin components on the surface of TI is the same as the momentum relaxation time $\left.{ }^{10}\right)$.

Although the $g$-factor in the Zeeman term $-g_{\|} \mu_{B} B_{x} \hat{\sigma}_{x}$ introduced by the external magnetic field applied parallel to the top and bottom surfaces of $\mathrm{Bi}_{2} \mathrm{Se}_{3}$ is renormalized ${ }^{7} g_{\|}=23$ due to strong SOC effects, ${ }^{27}$ changing its sign $B_{x} \rightarrow-B_{x}$ has virtually no effect on the spinpolarization vector governed by the strong surface SOC. For example, $P_{x}=0.605$ (at $E_{F}=3.04 \mathrm{eV}$ selected for illustration) in zero magnetic field $B_{x}=0$ is virtually indistinguishable from $P_{x}=0.609$ at large external magnetic field $B_{x}=-10 \mathrm{~T}$ applied opposite to the direction of electron transport. Thus, the same mechanismmomentum-dependent effective magnetic field associated with SOC which is much stronger than any external one - can be invoked to explain why $V_{\text {ISHE }}$ did not change sign upon reversing $B_{x} \rightarrow-B_{x}$ in the experiment of 

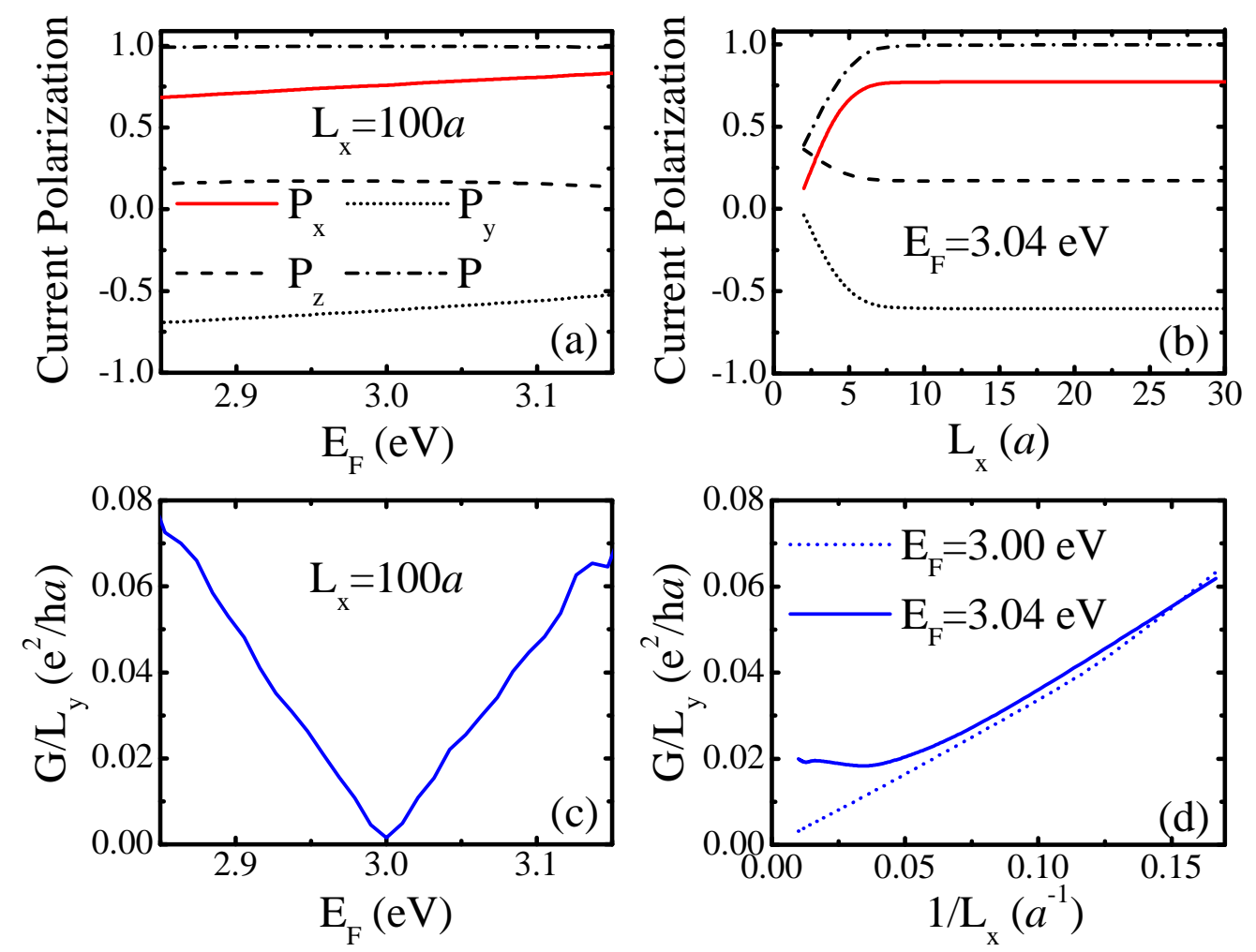

FIG. 3: (Color online) The components $\left(P_{x}, P_{y}, P_{z}\right)$ and the magnitude $|\mathbf{P}|$ of the spin-polarization vector of charge current outflowing into lead 2, after unpolarized charge current is injected from lead 1 into the two-terminal version of junction in Fig. 1 . These are plotted vs. (a) the Fermi energy $E_{F}$, or (b) the length $L_{x}$ of the TI film. The linear response charge conductance of the same two-terminal junction vs. (c) the Fermi energy, or (d) the inverse length $1 / L_{x}$.

Ref. 5. This requires that 2D hole gas (see Fig. 1 in Ref. 5 ) formed at the interface between Pt probe and InSb has SOC with a component of its effective magnetic field pointing in the direction of transport (as it would be the case in the presence of the Dresselhaus SOC7).

At first sight, the surface of 3D TI is expected to spin-polarize charge current in the transverse direction only, 10 13 P $P_{y} \neq 0$ while $P_{x}=P_{z}=0$. This is due to the fact that Dirac cone energy-momentum dispersion on the surface of TI and spin-orthogonal-to-momentum locking within it, as observed in spin-ARPES experiments,$[6$ is routinely described by an effective 2D Hamiltonian $\sqrt{625}$ taking form of the massless Rashba model, 7 $\hat{H}=v_{F}(\hat{\boldsymbol{\sigma}} \times \hat{\mathbf{p}}) \cdot \mathbf{e}_{z}$. Here $v_{F}$ is the Fermi velocity, $\hat{\mathbf{p}}=\left(\hat{p}_{x}, \hat{p}_{y}\right)$ is the momentum operator in $2 \mathrm{D}$ and $\hat{\boldsymbol{\sigma}}=\left(\hat{\sigma}_{x}, \hat{\sigma}_{y}, \hat{\sigma}_{z}\right)$ is the vector of the Pauli matrices. Thus, when charge current flows on the TI surface longitudinally, this Hamiltonian predicts induction of nonequilibrium transverse spin density 10 12] $S_{y}$ and the corresponding spin-polarization $P_{y} \neq 0$ of the current outflowing into the attached $\mathrm{N}$ leads $\frac{13}{13}$ This effect is the counterpart of the one predicted long ago, $\frac{1415}{115}$ and observed in recent experiments, $\frac{16}{16}$ for the Rashba spin-split 2DEGs, except that $S_{y}$ on the TI surface is larger by a factor $\hbar v_{F} / \alpha \gg 1$ (the Rashba SOC term in 2DEGs is given $\left.\operatorname{by}^{7} \alpha(\hat{\boldsymbol{\sigma}} \times \hat{\mathbf{p}}) \cdot \mathbf{e}_{z} / \hbar\right)$.

However, such conclusion is an artifact of a naïve identification of $\hat{\boldsymbol{\sigma}}$ operator with the true electron spin, which becomes invalid when the TI surface does not coincide with the QL plane ${ }^{26}$ Since low-energy Hamiltonian models ${ }^{25}$ of $\mathrm{Bi}_{2} \mathrm{Se}_{3}$ operate with the pseudospin degree of freedom describing states with support on the $\mathrm{Bi}$ and $\mathrm{Se}$ sublattices, the attachment of the TI thin film to semiinfinite $\mathrm{N}$ leads that inject or absorb electrons carrying real spin requires to properly interpret their mutual coupling when studying spin-dependent electron transport. Aligning QLs of $\mathrm{Bi}_{2} \mathrm{Se}_{3}$ perpendicularly to the infinite TI surface in $x y$-plane, and at an angle of $45^{\circ}$ with respect to the $y z$-plane, will generate difference in spin textures on the two sublattices shown in Fig. 2. This motivates our proposal for the junction setup in Fig. 1 where longitudinal spin polarization $\left[P_{x} \neq 0\right.$ in Fig. $3(\mathrm{a})$ and (b)] is driven by the surface-state electrons on the Se sublattice. This emerges in addition to the amply studied (in the diffusive $e^{10[12]}$ or in the ballistic ${ }^{13}$ transport regimes) transverse nonequilibrium spin density and polarization $\left[P_{y} \neq 0\right.$ in Fig. [3(a) and (b)] that is predominantly generated by the Bi sublattice. Note that $P_{z}$ component in Fig. 3(a) and (b) remains non-zero even if both surfaces are open for transport, or if the cross section in the $y z$ - 

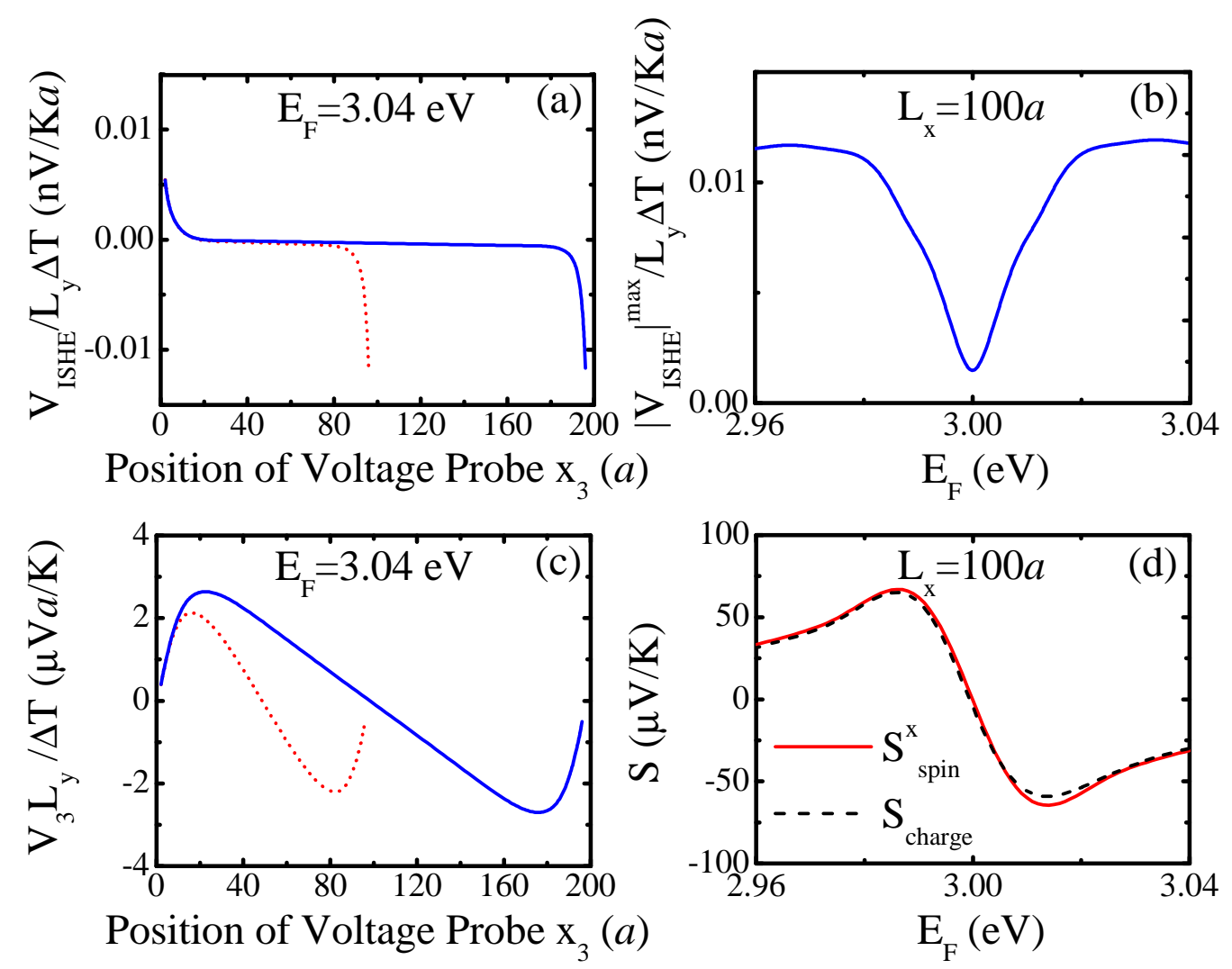

FIG. 4: (Color online) (a) The SSE signal $V_{\mathrm{ISHE}} /\left(L_{y} \Delta T\right)$ as a function of the position of $\mathrm{N}$ lead 3 in Fig. 1 displaced between the contacts with $\mathrm{N}$ leads 1 and 2 sandwiching TI samples of length $L_{x}=100 a$ or $L_{x}=200 a$. (b) Dependence of $\left|V_{\mathrm{ISHE}}\right|^{\max } /\left(L_{y} \Delta T\right)$ on the Fermi energy $E_{F}$. (c) Voltage $V_{3} L_{y} / \Delta T$ applied to $\mathrm{N}$ lead 3 in order to bring the net charge current $I_{3} \equiv 0$. (d) Conventional charge and spin-dependent Seebeck coefficients vs. $E_{F}$ for the two-terminal version of junction in Fig. 1 .

plane becomes infinite, since it originates from electrons tunneling through the bulk of the TI.

Figure 3(c) plots the linear-response conductance $G=\lim _{V_{b} \rightarrow 0} I_{2} / V_{b}$ of the TI thin film attached to two $\mathrm{N}$ leads as a function of the Fermi energy $E_{F}$ when small bias voltage $V_{b}=V_{1}-V_{2}$ drives charge current $I_{2}$. The $G$ vs. $E_{F}$ dependence exhibits a V-shape (slightly asymmetric due to the attached $\mathrm{N}$ leads) familiar from graphene, ${ }^{28129}$ with a minimum conductivity $\sigma=G L_{x} / L_{y}$ reached at the DP located at $E_{F}=3.0 \mathrm{eV}$. Even though the density of states vanishes at the DP, so that $\sigma$ should apparently approach zero at the DP, for this ballistic junction it remains non-zero due to evanescent wavefunctions injected by the metallic $\mathrm{N}$ leads. While they are similar to the well-known metal induced gap states in metal-semiconductor junctions, such states typically penetrate only a few atomic lengths into the semiconductor where the depth of penetration decreases with increasing band gap. On the other hand, evanescent states in N/TI junctions penetrate a much longer distance due to zero energy gap at the DP, as observed also in $\mathrm{N}$ /graphene junctions ${ }^{30}$ Figure 3(d) shows accidental $(\beta \approx 1)$ Ohmic scaling $G \propto L_{y} / L_{x}^{\beta}$ (for $\left.L_{y} / L_{x} \gg 1\right)$ at $\mathrm{DP}$, so that evanescent mode quantum transport in N/TI or $\mathrm{N} /$ graphene junctions is termed 29 "pseudo-diffusive."

\section{VOLTAGE SIGNAL OF SSE IN THREE-TERMINAL TI-BASED JUNCTIONS}

When the third $\mathrm{N}$ lead, assumed to be made of a heavy metal with sufficiently large ${ }^{31} \mathrm{SH}$ angle $\theta_{\mathrm{SH}}$, is attached to the top surface of the TI, as shown in Fig. 1, spin current $I_{3}^{S_{\alpha}}$ will be injected into it. Besides using temperature bias $\Delta T=T_{1}-T_{2}=2 \mathrm{~K}$ at average temperature $T=\left(T_{1}+T_{2}\right) / 2=50 \mathrm{~K}$ to drive $\mathrm{SSE}$, we also apply voltage $V_{3}$ to the macroscopic reservoir (attached to $\mathrm{N}$ lead 3 at infinity) in order to ensure that net charge current through it remains zero $I_{3} \equiv 0$ and $I_{3}^{S_{\alpha}} \neq 0$ is pure. The profile of $V_{3}$ across the TI thin film is plotted in Fig. 4(c). We assume that the reservoir temperature $T_{3}(x)=T_{1}-x\left(T_{1}-T_{2}\right) / L_{x}$ decreases linearly as $\mathrm{N}$ lead 3 is displaced between the contacts of TI film with $\mathrm{N}$ lead 1 and 2.

The central quantity in the theories ${ }^{4}$ of transverse SSE is $I_{3}^{S_{x}}$ component of the pure spin current injected into $\mathrm{N}$ lead 3 , which we compute per lattice spacing $a$ because of assumed periodicity of system in Fig. 1 in 
the $y$-direction. Since $I_{3}^{S_{x}}$ carries spins pointing along the $x$-axis, the ISHE mechanism illustrated in Fig. 1 will accumulate charges on the opposite edges of $\mathrm{N}$ lead 3 in the transverse direction. These generate electric field $\mathbf{E}_{\text {ISHE }}$ and the corresponding voltage signal ${ }^{4}$ $V_{\mathrm{ISHE}}=E_{\mathrm{ISHE}}^{y} L_{y}=\theta_{\mathrm{SH}}^{\mathrm{Pt}} I_{3}^{S_{x}} e \rho^{\mathrm{Pt}} / W$. To facilitate comparison with experiments, ${ }^{315}$ Fig. 4 plots $V_{\mathrm{ISHE}} /\left(L_{y} \Delta T\right)$ which has the same unit (after multiplying the results in Fig. 4 by $L_{y}$ ) as the conventional charge-Seebeck coefficient $S_{\text {charge }}=-\left(V_{1}-V_{2}\right) /\left(T_{1}-T_{2}\right)$ measured ${ }^{28}$ on two-terminal junctions. For this purpose, we assume that $I_{3}^{S_{x}}$ is converted into $V_{\text {ISHE }}$ via the ISHE operating within $\mathrm{N}$ lead 3 of width $W=6 a$ which is made of $\mathrm{Pt}$ with resistivity $\rho^{\mathrm{Pt}}=105 \mathrm{n} \Omega \mathrm{m}$ and with putative ${ }^{31} \mathrm{SH}$ angle $\theta_{\mathrm{SH}}^{\mathrm{Pt}}=0.08$.

The dependence of $V_{\text {ISHE }} /\left(L_{y} \Delta T\right)$ on the position of $\mathrm{N}$ lead 3 shown in Fig. 4(a) is asymmetric, thereby exhibiting a fundamental property of the transverse SSE where its signal changes sign between cold and hot ends of the sample. ${ }^{21315}$ The maximum signal $\left|V_{\mathrm{ISHE}}\right|^{\max } /\left(L_{y} \Delta T\right)$ is reached around the sample edges and it is independent of length $L_{x}$, as shown in Fig. 4(a). Unlike the spindependent Seebeck coefficient $S_{\text {spin }}^{x}$ plotted in Fig. 4 (d), which quantifies spin current injected into $\mathrm{N}$ lead 2 due to temperature bias applied to the two-terminal version of junction in Fig. 1. $\left|V_{\text {ISHE }}\right|^{\max } /\left(L_{y} \Delta T\right)$ vs. $E_{F}$ within the bulk gap of TI plotted in Fig. 4(b) is unrelated to conventional CS coefficient $S_{\text {charge }}$. Note that both $S_{\text {spin }}^{x}$ and $S_{\text {charge }}$ in Fig. 4(d) exhibit the same dependence on $E_{F}$ as $S_{\text {charge }}$ measured on graphene. ${ }^{28}$ This is due to the fact that electron- and hole-like transport gives contributions to these coefficients of opposite sign, so that $S_{\text {spin }}^{x}=S_{\text {charge }} \equiv 0$ exactly at the DP while reaching maximum absolute value few $k_{B} T$ away from it.

\section{CONCLUDING REMARKS}

In conclusion, we predict that thermally driven charge current on the surface of 3D TI thin film, realized us- ing $\mathrm{Bi}_{2} \mathrm{Se}_{3}$ whose QLs are oriented at an angle of $45^{\circ}$ with respect to the direction of transport while being perpendicular to the TI surface (see Fig. 1), will become spin-polarized due to strong surface SOC. In addition to amply studied $d^{10}[13$ nonequilibrium transverse spin polarization on the TI surface parallel to QLs, for the orientation of QLs we propose in Fig. 1 the spin-polarization vector will acquire an additional component parallel to the direction of charge transport which is generated by the Se sublattice. This makes it possible to recreate the phenomenology of electron-driven SSE recently observed in InSb $\sqrt[5]{5}$ but in the absence of any external magnetic field. The predicted magnitude of SSE signal shown in Figs. 4(a) and 4(b) can be translated into experimentally measurable voltage by multiplying it with $L_{y}$ and $\Delta T$, e.g., $\left|V_{\mathrm{ISHE}}\right|^{\max } \simeq 5 \mu \mathrm{V}$ if we assume $L_{y}=1 \mathrm{~mm}$, $\Delta T=2 \mathrm{~K}$ and $a \approx 3.4 \mathrm{~nm}$ (which is the effective lattice constant of our simple cubic lattice for the distance $c \approx 2.9 \mathrm{~nm}$ between QLs oriented as in Fig. 11). We believe that this value could be further enhanced by including phonon-electron drag effect, speculated to play a key role in achieving the "giant" magnitude of SSE measured in Ref. 5, which we relegate to future studies.

\section{Acknowledgments}

We thank E. Saitoh for illuminating discussions. P.H. C., F. M. and B. K. N. were supported by NSF under Grant No. ECCS 1202069. N. N. was supported by Grant-in-Aids for Scientific Research (21244053) from the Ministry of Education, Culture, Sports, Science and Technology of Japan, Strategic International Cooperative Program (Joint Research Type) from Japan Science and Technology Agency, and also by Funding Program for World-Leading Innovative R\&D on Science and Technology (FIRST Program).
* Electronic address: bnikolic@udel.edu

${ }^{1}$ G. E. W. Bauer, E. Saitoh, and B. J. van Wees, Nature Mater. 11, 391 (2012).

${ }^{2}$ K. Uchida, T. Ota, H. Adachi, J. Xiao, T. Nonaka, Y. Kajiwara, G. E. W. Bauer, S. Maekawa, and E. Saitoh, J. Appl. Phys. 111, 103903 (2012).

3 C. M. Jaworski, J. Yang, S. Mack, D. D. Awschalom, J. P. Heremans, and R. C. Myers, Nature Mater. 9, 898 (2010).

${ }^{4}$ H. Adachi, K. Uchida, E. Saitoh, and S. Maekawa, Rep. Prog. Phys. 76, 036501 (2013).

${ }^{5}$ C. M. Jaworski, R. C. Myers, E. Johnston-Halperin, and J. P. Heremans, Nature 487, 210 (2012); T. T. Heikkilä and Y. Tserkovnyak, Nature 487, 180 (2012).

6 M. Z. Hasan and C. L. Kane, Rev. Mod. Phys. 82, 3045 (2010); X.-L. Qi and S.-C. Zhang, Rev. Mod. Phys. 83 1057 (2011).
7 R. Winkler, Spin-Orbit Coupling Effects in TwoDimensional Electron and Hole Systems (Springer, Berlin, 2003).

${ }^{8}$ D. Kim, S. Cho, N. P. Butch, P. Syers, K. Kirshenbaum, S. Adam, J. Paglione, and M. S. Fuhrer, Nature Phys. 8, 459 (2012).

${ }^{9}$ W. Luo and X.-L. Qi, Phys. Rev. B 87, 085431 (2013).

10 A. A. Burkov and D. G. Hawthorn, Phys. Rev. Lett. 105, 066802 (2010).

11 T. Misawa, T. Yokoyama, and S. Murakami, Phys. Rev. B 84, 165407 (2011).

12 D. Pesin and A. H. MacDonald, Nature Mater. 11, 409 (2012).

13 S. Modak, K. Sengupta, and D. Sen, Phys. Rev. B 86, 205114 (2012)

14 V. M. Edelstein, Solid State Comm. 73, 233 (1990). 
15 J. I. Inoue, G. E. W. Bauer, and L. W. Molenkamp, Phys. Rev. B 67, 033104 (2003).

16 S. D. Ganichev, S. N. Danilov, Petra Schneider, V.V. Bel'kov, L. E. Golub, W. Wegscheider, D. Weiss, and W. Prettl, J. Magn. Magn. Mater 300, 127 (2006).

17 G. Stefanucci and R. van Leeuwen, Nonequilibrium ManyBody Theory of Quantum Systems: A Modern Introduction (Cambridge University Press, Cambridge, 2013).

18 M. Scheid, D. Bercioux, and K. Richter, New J. Phys. 9, 401 (2007).

19 B. K. Nikolić, L. P. Zârbo, and S. Souma, Phys. Rev. B 72, 075361 (2005).

20 S. Datta, Electronic transport in mesoscopic systems (Cambridge University Press, Cambridge, 1995).

21 T. P. Pareek, Phys. Rev. Lett. 92, 076601 (2004).

${ }^{22}$ U. Sivan and Y. Imry, Phys. Rev. B 33, 551 (1986).

23 B. K. Nikolić, K. K. Saha, T. Markussen, and K. S. Thygesen, J. Comp. Electron. 11, 78 (2012).

${ }^{24}$ K. Park, J. J. Heremans, V. W. Scarola, and D. Minić,
Phys. Rev. Lett. 105, 186801 (2010); O. V. Yazyev, J. E. Moore, and S. G. Louie, Phys. Rev. Lett. 105, 266806 (2010).

25 C.-X. Liu, X.-L. Qi, H. J. Zhang, X. Dai, Z. Fang, and S.-C. Zhang, Phys. Rev. B 82, 045122 (2010).

26 P. G. Silvestrov, P. W. Brouwer, and E. G. Mishchenko, Phys. Rev. B 86, 075302 (2012); F. Zhang, C. L. Kane, and E. J. Mele, Phys. Rev. B 86, 081303 (2012).

27 H. Köhler and E. Wöchner, Phys. Status Solidi B 67, 665 (1975).

28 Y. M. Zuev, W. Chang, and P. Kim, Phys. Rev. Lett. 102, 096807 (2009).

29 J. Tworzydło, B. Trauzettel, M. Titov, A. Rycerz, and C. W. J. Beenakker, Phys. Rev. Lett. 96, 246802 (2006).

${ }^{30}$ R. Golizadeh-Mojarad and S. Datta, Phys. Rev. B 79, 085410 (2009).

31 L. Liu, R. A. Buhrman, and D. C. Ralph, arXiv:1111.3702 